\title{
Situación organizacional de los Grupos de Control de Vectores en Ciudad de La Habana
}

\section{Organizational situation of Vector Control Groups in the City of Havana}

\author{
Vivian Noriega Bravo'; I sora Ramos Valle'; Lourdes Couterejuzón \\ González"; Libertad Martín Alfonso" "'; Magdalena Mirabal Jean-Claude' ${ }^{\prime V}$; \\ Guillermo Díaz Llanes ${ }^{\prime \prime \prime}$ \\ 'Especialista de II Grado en Epidemiología. Máster en Salud Pública. Escuela \\ Nacional de Salud Pública. La Habana, Cuba. \\ "Especialista de II Grado en Bioestadística. Máster en Informática Médica. Escuela \\ Nacional de Salud Pública. La Habana, Cuba. \\ "'Licenciado en Psicología. Máster en Psicología de la Salud. Escuela Nacional de \\ Salud Pública. La Habana, Cuba. \\ ${ }^{1 V}$ Especialista de II Grado en Higiene Escolar. Máster en Salud Pública. Escuela \\ Nacional de Salud Pública. La Habana, Cuba.
}

\section{RESUMEN}

I ntroducción Se desarrolló una investigación operativa en octubre de 2006, que en breve tiempo aportó información útil para la toma de decisiones en el trabajo de erradicación del mosquito Aedes aegypti.

Objetivos Caracterizar la situación organizacional existente en los Grupos de Control de Vectores de la provincia Ciudad de La Habana, la llamada "Campaña" e identificar aquellos factores que obstaculizaban el trabajo.

Métodos Se realizó un estudio descriptivo y transversal. Se trabajó con una muestra intencional que incluyó 278 trabajadores de diferentes categorías ocupacionales y procedentes de 15 áreas de salud, seleccionadas a partir de una estratificación de la provincia por criterio cualitativo. Se estudiaron también 57 directivos. Se exploraron las variables tiempo en la actividad y otras relacionadas con el propósito de la organización, estructura organizativa, capacitación, relaciones entre trabajadores y con los superiores en rango (jefes), dentro y fuera del sector; 
mecanismos de apoyo, uso y formas de reconocimiento, condiciones de trabajo y recursos. Además, se evaluó el clima organizacional a partir de 16 criterios en las dimensiones: Liderazgo, Motivación, Reciprocidad y Participación. Se emplearon técnicas cuantitativas y cualitativas: encuesta, entrevista a profundidad y grupo focal, y se trianguló la información obtenida.

Resultados Los principales resultados estuvieron en la inadecuada selección del personal y poco tiempo en la actividad, insuficiente integración de los operarios al trabajo del Equipo Básico de Salud no inherente a la estructura organizativa, inadecuadas normas de trabajo que propiciaban conductas fraudulentas, insuficiencias en los mecanismos de apoyo, de estimulación y reconocimiento que generaban desmotivación en los trabajadores; estilos de dirección que dificultaban la comunicación y las relaciones interpersonales y métodos de dirección poco participativos.

Conclusiones Se localizan numerosos y diversos problemas de tipo organizacional que influyen desfavorablemente en el clima laboral y en consecuencia limitan la dinámica de trabajo en los grupos estudiados.

Palabras claves: Situación organizacional, elementos organizacionales, clima organizacional, Grupos de Control de Vectores.

\section{ABSTRACT}

Introduction An operational research was made in October, 1996 that quickly offered useful information for decision-making on the work carried out to eradicate Aedes aegypti.

Objectives: To characterize the organizational situation of Vector Control Groups in the City of Havana province and identify those factors that hinder its work.

Methods A descriptive cross-sectional study that uses an intentional sample of 278 workers of various occupational categories from 15 health areas that have been previously selected on the basis of stratification by qualitative criteria. Fifty seven directors were also included. Variables such as length of time performing this activity and others related to the objective of the organization; organizational structure, training, relationship between workers and higher rank staff (heads) within and outside the sector, supporting mechanisms, use and ways of acknowledgement, working conditions and resources were examined. Additionally, the organizational environment based on 16 criteria in dimensions, i.e., leadership, motivation, reciprocity and participation. Quantitative and qualitative techniques were used like survey, in-depth interview, and focal groups. Obtained information was triangled.

Results The main results were the poor selection of the staff and short length of time in the activity; low integration of workers to the Basic Health Team noninherent to the organizational structure, inappropriate working standards that facilitate fraud, deficiencies found in the supporting, stimulation and acknowledgement mechanisms, thus discouraging workers, management methods that make it difficult to communicate each others, and inter-personal relations; management methods with low participation.

Conclusions Many diverse organizational problems were found. They unfavorably influence the working environment, and consequently restrict the work dynamics of the studied group.

Key words: Organizational situation, organization elements, organizational climate, vector control groups. 


\section{NTRODUCCIÓN}

Si bien antes del triunfo revolucionario de 1959 se ejecutaba en Cuba una estrategia contra el mosquito Aedes aegypti a través del funcionamiento de una estructura organizativa, que actuando como departamento ejecutivo autónomo y asesorado por la Organización Panamericana de la Salud, desarrollaba un plan de acción dirigido a erradicar el vector transmisor de la fiebre amarilla y el dengue, primero territorialmente y luego con acciones integradas a los servicios generales de salud, ${ }^{1}$ no es hasta 1981, después de la epidemia de dengue hemorrágico, que se consolida en el país una estructura organizativa y funcional para la actividad de vigilancia y lucha antivectorial, la denominada Campaña Nacional de Erradicación del Aedes aegypti y se implementa además un sólido programa de trabajo.

En correspondencia con las transformaciones llevadas a cabo en el marco del proceso de descentralización y desarrollo del nivel local en los años 90 , se crearon las subdirecciones de Higiene y Epidemiología en cada policlínico, con lo que se asumió una nueva dimensión de trabajo por la necesidad de acercar las decisiones al lugar donde se producían los problemas de salud. Con ello se estructuraron los Grupos de Control de Vectores, organizado en brigadas y por Consejos Populares y que en sus inicios respondieron técnica y administrativa a los Centros y Unidades Municipales de Higiene y Epidemiología (C-UMHE). ${ }^{2}$

Años más tarde, se establece el funcionamiento de esta estructura organizativa subordinada administrativamente al Área de Higiene y Epidemiología de los policlínicos y atendidos metodológicamente por las Unidades Municipales de Vigilancia y Lucha Antivectorial (UMVLA). En el organigrama se dispone la existencia de un jefe de Área de Vectores al que responden en orden jerárquico: supervisores de la Campaña, jefes de brigadas y operarios. ${ }^{3}$

Este personal se agrupa en brigadas, cuyo número varía según la cantidad de locales existente en el área, de la productividad que debe tener el operario en un día de trabajo y del tiempo de duración de la fase o ciclo, que no es más que el período establecido para garantizar la oportunidad de las acciones según el ciclo vital del mosquito. Las brigadas conformadas por operarios "A"u operarias "B", según sea el caso, realizan un trabajo integral en control de vectores hasta el nivel de consultorio.

Pese a que en Cuba se desarrolla una Estrategia Integrada para la Prevención y Control del dengue, altamente priorizada, en los últimos años algunas provincias, entre ellas Ciudad de La Habana, no han logrado mantener de forma estable índices bajos de infestación por Aedes aegypti, y en consecuencia, han ocurrido brotes epidémicos de la enfermedad, como el sucedido en el segundo semestre de 2006.

Este evento promovió no pocos criterios negativos del quehacer de los Grupos de Control de Vectores, por lo que fue interés de la dirección del Ministerio de Salud Pública conocer cuál era la situación existente, en el orden organizacional y qué factores estaban obstaculizando el trabajo; en respuesta a un encargo, la Escuela de Salud Pública (ENSAP) desarrolló una investigación que de manera rápida 
proveyó de información útil para la toma de decisiones. Sus resultados se exponen en el presente trabajo.

\section{MÉTODOS}

Se realizó un estudio descriptivo y transversal en áreas de salud seleccionadas de la provincia Ciudad de La Habana, en el mes de octubre de 2006.

El universo de estudio estuvo constituido por el total de trabajadores que laboraba en la Campaña en las áreas de salud de la provincia, además de los directivos según los cargos de: directores municipales de salud, de UMVLA y C-UMHE; directores y vicedirectores de Higiene y Epidemiología de las áreas de salud; y jefes municipales del Programa de Erradicación del Aedes aegypti.

Considerando la factibilidad en cuanto a recursos y tiempo, se seleccionó una muestra no probabilística de áreas de salud representativas de los 15 municipios con que cuenta la provincia. Para ello se conformaron dos estratos tomando como criterio los índices de infestación del vector reportados por la Unidad Provincial de Vigilancia y Lucha Antivectorial hasta el 9 de septiembre y se estableció un punto de corte en el índice con valor de 0,073 \%. El estrato I lo conformaron los municipios con índice igual o superior a este valor y el estrato II los de índice por debajo de $0,073 \%$.

Se escogió un área de salud por cada municipio teniendo en cuenta también el índice de infestación reportado. En el estrato I se seleccionaron por cada municipio las áreas de salud con mayor índice reportado, mientras que en el estrato II se eligieron aquellas áreas que maximizaran la condición de menor infestación. De esta manera las áreas objeto de estudio fueron:

Estrato I: Ana Betancourt (Playa), Puentes Grandes (Plaza de la Revolución), Reina (Centro Habana), Robert M. Zulueta (Habana Vieja), Luis Pasteur (10 de octubre), Lidia y Clodomira (Regla), Gregorio Valdés (Habana del Este), Mulgoba (Boyeros) y Abel Santamaría (Cerro).

Estrato II: California (San Miguel del Padrón), Ramón González Coro (Marianao), Pedro Fonseca (La Lisa), Párraga (Arroyo Naranjo), Cuatro Caminos (Cotorro) y Docente (Guanabacoa).

Se realizó un muestreo intencional de trabajadores en las diferentes categorías ocupacionales por cada área de salud: 1 jefe de área, 5 supervisores, 10 jefes de brigada "A"o "B, 6 operarios "A"y 6 operarias "B". Los criterios de inclusión fueron: llevar un año o más de labor en la Campaña en el área de salud donde se estaba ejecutando la investigación y haber expresado verbalmente su consentimiento para participar del estudio. Finalmente la muestra incluyó a 278 trabajadores.

Se exploraron variables relacionadas con el tiempo en la actividad, propósito de la organización, estructura organizativa, capacitación, relaciones entre trabajadores y con los superiores en rango (jefes), dentro y fuera del sector; mecanismos de apoyo, uso y formas de reconocimiento, condiciones de trabajo y recursos y clima laboral. 
Para recopilar la información se empleó un cuestionario, aplicado por entrevistadores ( 28 estudiantes de la maestría de Salud Pública versión 2006-08) previamente adiestrados, compuesto de 36 preguntas, dicotómicas, politómicas y abiertas. Este cuestionario, utilizado en investigaciones anteriores de la ENSAP, fue adecuado al objeto de estudio y validado su contenido en consulta con profesores y expertos en la actividad (funcionarios y jefe provincial del Programa).

Para indagar acerca de elementos que caracterizaban al clima organizacional, se empleó un cuestionario autoadministrado (Inventario de clima), también validado y utilizado en investigaciones anteriores; conformado por 48 afirmaciones con las que se exploraron cuatro dimensiones o "áreas críticas", según las define en su metodología para Diagnóstico Organizacional la Dra. Leonor Jiménez Canga: ${ }^{4}$ Liderazgo, Motivación, Reciprocidad y Participación; y en cada una de ellas se examinaron cuatro criterios: Dirección, Estímulo a la excelencia, Estímulo al trabajo en equipo, Solución de conflictos, Realización personal, Reconocimiento a la aportación, Responsabilidad, Adecuación a las condiciones de trabajo, Aplicación del trabajo, Pertenencia institucional, Retribución, Equidad, Compromiso con la productividad, Compatibilidad de intereses, Intercambio de la información e Involucración con el cambio.

Los encuestados valoraron de verdadero (V) o falso (F) cada uno de estos enunciados, según se aplicaran o no a su ambiente de trabajo.

Para el procesamiento se recurrió a una plantilla, en la que aparecían calificados cada uno de ellos como V o F. Se otorgó valor de 1 cuando existió coincidencia entre la respuesta dada por el encuestado y la calificación prevista, de lo contrario se otorgó el valor cero. Posteriormente se procedió a realizar la sumatoria de todos los puntos obtenidos en cada afirmación ( 3 por cada criterio) y se dividió por el total de personas que llenaron el instrumento. La evaluación de cada criterio se alcanzó con la suma de las cifras obtenidas en el paso anterior y cuyos valores estuvieron entre 0 y 3 . Se estableció como punto de corte o estándar, el valor de 2,1 . Las cifras que resultaron por debajo de este se valoraron de insatisfactorias y en ese caso se consideró que la dimensión explorada estaba afectando negativamente el clima laboral. Por último, se evaluó el clima en favorable o desfavorable, si 2 o más de las dimensiones estudiadas tuvieron sobre este elemento una influencia negativa.

Con el interés de obtener otras valoraciones acerca de la estructura organizativa, uso de la información, mecanismos de cooperación, así como las principales dificultades para el cumplimiento de las actividades; se realizaron entrevistas a profundidad a los directivos que estuvieron disponibles en el momento de la investigación; en total 57.

También, a fin de ahondar y clarificar puntos de vista expresados por los encuestados, se realizaron grupos focales en cinco de las áreas de salud correspondiente al estrato I: Abel Santamaría, Reina, Ana Betancourt, Puentes Grandes, Luis Pasteur y en el área Camilo Cienfuegos (segunda más alta en índice de infestación reportada por el municipio Habana del Este).

El conocimiento del número total de informantes claves por cada área permitió ejecutar un muestreo intencional que determinó la cantidad de grupos focales y la selección de los participantes; finalmente se escogieron de modo aleatorio hasta 12 o 15 informantes claves para el desarrollo de las sesiones. Se realizaron grupos homogéneos con operarios "A" $y$ operarias "B" $y$ heterogéneos con jefes de brigadas y supervisores, por ser estos últimos los que controlan el proceso de 
trabajo y los primeros ejecutores de las acciones. En total se efectuaron 10 grupos focales en los que participaron 74 trabajadores, de ellos 56 hombres y 18 mujeres, en las categorías de jefe de área (1), supervisor (10), jefe de brigada (20) y operario (43).

Para la realización de los grupos cada sesión contó con un moderador, un relator y un observador, con funciones metodológicas específicas, en este caso fueron estudiantes de la maestría de Psicología de la Salud debidamente capacitados en las habilidades necesarias para el desarrollo de la técnica y el análisis de la información generada en las sesiones de discusión.

Se realizó al menos una sesión de trabajo de aproximadamente una hora y media de intercambio, tiempo suficiente para que los grupos produjeran la información necesaria. Se elaboraron las preguntas llevadas a la discusión grupal atendiendo al objetivo propuesto para la tarea de investigación.

Para asegurar que el diseño aportara el resultado esperado y hacer los ajustes pertinentes, se realizó previamente un pilotaje de la metodología propuesta en un área de salud no seleccionada para el estudio.

Con la información obtenida se realizó un análisis de contenidos, se agrupó esta en categorías y en cada una de ellas se establecieron tipologías o grupos teniendo en cuenta los criterios de percepción de los participantes.

\section{RESULTADOS}

Como se aprecia en la tabla 1, la mayoría de los trabajadores de todas las categorías llevaban entre 1 y 3 años de labor en la Campaña y ninguno de los encuestados sobrepasaba los 10 años. Vale significar la elevada proporción en que se presenta esta variable tanto en operarios como operarias. Similar comportamiento se observó en los directivos, en los que el 29,8 \% tenía menos de 1 año desempeñando tareas vinculadas con la actividad.

Tabla 1. Distribución de directivos y trabajadores por categorías según tiempo en la actividad que realizan

\begin{tabular}{|c|c|c|c|c|c|c|c|c|c|c|c|c|}
\hline \multirow{2}{*}{$\begin{array}{l}\text { Tiempo } \\
\text { (años) }\end{array}$} & \multicolumn{2}{|c|}{ J'área } & \multicolumn{2}{|c|}{ Supervisor } & \multicolumn{2}{|c|}{ J' brigada } & \multicolumn{2}{|c|}{ Operario "A" } & \multicolumn{2}{|c|}{ Operaria "B" } & \multicolumn{2}{|c|}{ Directivo } \\
\hline & No. & $\%$ & No. & 9 & No. & $\%$ & No. & $\%$ & No. & $\%$ & No. & $\%$ \\
\hline $\begin{array}{l}\text { Menos } \\
\text { de } 1\end{array}$ & & & & & & & & & & & 17 & 29,8 \\
\hline $1-3$ & 5 & 83,3 & 18 & 64,2 & 66 & 84,6 & 82 & 94,3 & 73 & 92,4 & 24 & 42,1 \\
\hline $4-5$ & 1 & 16,7 & 10 & 35,8 & 11 & 14,1 & 4 & 4,6 & 3 & 3,8 & 5 & 8,9 \\
\hline $6-10$ & - & - & - & - & 1 & 1,3 & 1 & 1,1 & 3 & 3,8 & 4 & 7,0 \\
\hline Más 10 & - & - & - & - & - & - & - & - & - & - & 7 & 12,2 \\
\hline Total & 6 & 100,0 & 28 & 100,0 & 78 & 100,0 & 87 & 100,0 & 79 & 100,0 & 57 & 100,0 \\
\hline
\end{tabular}

Al explorar aspectos relacionados con los propósitos de la organización, se encontró que más de la mitad de los encuestados (64,3 \%) opinaron conocer la misión de la subdirección de Higiene y Epidemiología y esencialmente la declararon como "...la 
lucha antivectorial, control de las actividades de la Campaña, erradicación y control del mosquito Aedes aegypti y los vectores en general."

El conocimiento de las funciones fue referida por el $88 \%$ de los trabajadores y aunque semánticamente mezclaron funciones con tareas o actividades, se apreció dominio de las funciones que realizaban; el resto no conocían o conocían parcialmente las funciones de su departamento. Así mismo, el 92,4 \% dijo que existía total correspondencia de estas con las tareas y actividades que realizaban, mientras que un reducido número la consideró parcial.

Un elevado porcentaje de trabajadores consideró la idoneidad de la estructura organizativa establecida para el cumplimiento de las funciones y la facilidad que brindaba para la integración del operario al trabajo del Equipo Básico de Salud. Los que opinaron contrario refirieron cuestiones tales como:

...no existe tal integración, esta sólo existe cuando hay epidemia. El operario dedica todo el tiempo a buscar focos no se busca integración, solamente orientar el trabajo. No se nos da participación en las reuniones del Grupo Básico de Trabajo y tampoco se está informado de cómo participar. Existen pocos médicos y tienen mucha sobrecarga de trabajo por lo que no disponen de tiempo para atender el trabajo de la Campaña.

Se profundizó en este análisis a partir de la información dada por los participantes en los grupos focales, y en general, se consideró adecuada la cadena de mando: jefe de área-supervisor-jefe de brigada-operario, no obstante existir en algunos, inconformidad con la subordinación actual al área de salud.

La estabilidad del personal fue referida como un problema y más de la mitad $(65,5$ \%) expresó que el no completamiento de la plantilla interfería con el cumplimiento de las tareas. Los participantes en las discusiones grupales añadieron elementos de interés relacionados con la estructura:

... hay inadecuada selección del personal que además le resta prestigio al trabajo de la Campaña; hay inestabilidad de la fuerza de trabajo por poca estimulación, por sobrecarga en el cumplimiento de las normas, extensas jornadas de trabajo que dificulta hasta disfrutar de descanso y por la mala atención al hombre.

Casi la totalidad de los encuestados opinó que las actividades diarias le son orientadas por el jefe inmediato superior, aunque unos pocos refirieron sentirse dirigidos por muchas personas e incluso, recibir orientaciones directamente de los niveles superiores.

A la pregunta específica sobre la permanencia de los jefes en sus cargos, el $85 \%$ la valoró de Buena y el $11,5 \%$ de Regular.

La asignación del universo de trabajo fue valorado por muchos de Adecuada y aunque los comentarios al respecto fueron casi nulos, las opiniones emitidas en las sesiones de los grupos focales coincidieron en afirmar que las normas eran altas y que propiciaban conductas fraudulentas por la imposibilidad de ser cumplidas. No obstante, 7 de cada 10 encuestados expresaron que se cumplía con el universo asignado y algunos hasta refirieron sobrecumplimiento, argumentando que "... se tiene dominio del territorio y se emplean muchas horas extras de trabajo fundamentalmente cuando hay epidemia." 
Las actividades de control fueron valoradas de Muy útil por un 59,5\%, si se le suma a ello los que la catalogaron de Útil, entonces casi todos consideraron la utilidad del control, y en similar proporción, afirmaron que ello aporta al mejoramiento del trabajo. Aquellos que opinaron que este tipo de actividad era Poco útil, se refirieron a la mala organización y no sistematicidad de las revisiones y fiscalizaciones. También el análisis cualitativo demostró iguales consideraciones:

...existe excesiva supervisión por los diferentes directivos; las actividades de control están mal diseñadas y los métodos utilizados no son adecuados; hay excesivo control por parte de los niveles superiores lo cual crea un clima de desconfianza y rechazo hacia la tarea, no hay trabajo preventivo, ni sistematicidad en el control durante el resto del año.

Por su parte la mayoría de los directivos entrevistados expresaron que uno de los mayores problemas que tenía el trabajo de la Campaña era la debilidad de la actividad de control.

Casi todos los trabajadores opinaron haber sido preparados para las actividades que realizaban y un poco más de la mitad dijo haber recibido algún tipo de capacitación en el último año; no obstante existir criterios desfavorables en relación con este proceso, tanto en la capacitación para el empleo como para corregir los problemas del desempeño. En este sentido, sólo el 32,1\% de los encuestados consideró que Siempre recibió capacitación al concluir cada ciclo de trabajo (reciclaje), el resto refirió que Nunca o sólo A veces.

En opinión de los directivos, el nivel de preparación es deficiente y en consecuencia se afecta la calidad del trabajo. De igual manera, los jefes de brigadas, supervisores y operarios en los grupos focales, plantearon que "...antes se hacían cursos de hasta 6 meses, ahora duran entre 5 y 15 días por lo que la preparación es insuficiente y es necesaria para un mejor trabajo de la Campaña."

Al preguntar acerca de los espacios más efectivos para establecer las coordinaciones de trabajo tanto dentro como fuera del sector salud, se refirió como mejor el Puesto de Mando. Nótese en la tabla 2, cuan bajos resultaron los porcentajes dados a otros espacios que deben ser igualmente identificados como ventajosos para coordinar acciones conjuntas en la lucha contra el Aedes aegypti en la comunidad.

Tabla 2. Opinión de los encuestados acerca del espacio más efectivo para establecer coordinaciones de trabajo

\begin{tabular}{|l|c|c|c|c|}
\hline \multirow{2}{*}{ Espacios } & \multicolumn{3}{|c|}{$\begin{array}{c}\text { Opinión } \\
n=278\end{array}$} \\
\cline { 2 - 3 } & \multicolumn{2}{|c|}{ Si } & No \\
\hline Puesto de mando & No. & $\%$ & No. & $\%$ \\
\hline $\begin{array}{l}\text { Comunicación directa con representantes de } \\
\text { organizaciones de masas }\end{array}$ & 196 & 70,5 & 82 & 29,5 \\
\hline Reunión del Consejo Popular & 102 & 36,7 & 176 & 63,3 \\
\hline $\begin{array}{l}\text { Comunicación directa con representantes de } \\
\text { organizaciones políticas }\end{array}$ & 60 & 21,6 & 218 & 78,4 \\
\hline Reunión del GBT & 41 & 14,7 & 237 & 85,3 \\
\hline $\begin{array}{l}\text { Intercambio con el médico y enfermera de } \\
\text { familia }\end{array}$ & 37 & 13,3 & 241 & 86,7 \\
\hline
\end{tabular}


En este sentido, el 79,3\% de los trabajadores refirieron haber sido apoyados ante situaciones concretas surgidas en el trabajo diario y las valoraciones acerca de quien o de quienes han recibido el apoyo, señalaron con el mayor porcentaje de Adecuado a los compañeros de equipo $(90,9 \%)$, seguido de los jefes y en un bajo porcentaje se ubicaron a los sectores, organizaciones de masa: Comités de Defensa de la Revolución (CDR) y Federación de Mujeres Cubanas (FMC) y a la población.

Referido a la falta de apoyo dado por otros sectores, elemento que se reiteró en las entrevistas a los directivos, se planteó la falta de sostenibilidad de las acciones fuera de las situaciones de emergencia epidemiológica, además del déficit de recursos.

Se refirió por la mayoría de los participantes en el estudio, que el limitado apoyo que brindan las organizaciones de masas constituye uno de los principales factores que estaba afectando el trabajo de la Campaña en la capital. Entre las opiniones emitidas tanto en la encuesta como en los grupos focales estuvieron las siguientes "...poco cuidado de la higiene ambiental, desconocimiento de la labor del campañista y su importancia, trato irrespetuoso de la población hacia ellos". Aunque según la encuesta, sólo el 13 \% opinó que Nunca recibió de la población un trato respetuoso, el 66,7 \% expresó que En ocasiones.

En varias dinámicas de grupo se reconoció como favorable para el trabajo, el apoyo brindado por el Ejército Juvenil del Trabajo (EJT) y en algún momento la labor de los Trabajadores Sociales.

La relación jefe-subordinado, valorada por el $82,4 \%$ como Buena, tuvo entre las opiniones de los que la consideraron Regular o Mala las siguientes:

...no escuchan los problemas de los subordinados; existe imposición, ordeno y mando para todo, sólo se habla de expulsar del puesto de trabajo al que no cumple; es difícil verlos porque casi siempre están reunidos, discuten mucho y fuerte, generalmente están molestos y tensos, es muy limitada la comunicación sólo para asignar tareas; la comunicación es efectiva solamente cuando hay epidemia, no hay tiempo para relacionarse con los jefes, no se comunican ni para dar información de la situación existente.

De igual modo, 8 de cada 10 encuestados valoraron de Buena la comunicación con el nivel superior, el resto la consideró Regular o Mala porque:

...no llegan todas las informaciones del municipio, tienen en ocasiones formas inadecuadas para plantear los problemas, se dirigen a los trabajadores en tono amenazante y de regaño y no se escucha el criterio de los trabajadores.

Los grupos focales aportaron que las mejores relaciones se dan entre los trabajadores de igual cadena de mando, operario-operario. Las opiniones al respecto se concretaron en que "...la relación jefe-subordinado se caracteriza por un estilo autocrático, rígido, no educativo que afecta el trabajo y la satisfacción de los operarios."

Sobre la capacidad resolutiva de los jefes, más de la mitad de los encuestados la consideraron Adecuada, en tanto que el 32 \% la estimó entre Medianamente Adecuada e Inadecuada, refiriendo que las limitaciones para resolver los problemas obedecen al desconocimiento que se tiene de la actividad. 
Uno de los aspectos que aportó más opiniones negativas, fue lo relacionado con las condiciones de trabajo y recursos. La mayoría consideró Inadecuado o Medianamente Adecuado el ambiente físico de trabajo, pese a que poco más de la mitad de los encuestados percibía Agradable el ambiente laboral. Aquellos con criterio diferente argumentaron deficientes condiciones higiénico-sanitarias y confort.

En correspondencia con los criterios expuestos en las sesiones de los grupos focales, más de la mitad de los operarios, jefes de brigadas y supervisores encuestados, opinaron no contar de forma mantenida con todos los recursos materiales necesarios para el trabajo: mochilas aptas, medios de protección, modelajes, manuales de procedimientos y transporte para trasladarse hacia lugares distantes portando equipos de fumigación. También se obtuvo información acerca de irregularidades con el suministro de materiales para el aseo personal, uniformes, calzados y la alimentación. Resultó un criterio casi generalizado, que todas las dificultades en las condiciones de trabajo y recursos mejoran durante las emergencias epidemiológicas.

Muchos de los trabajadores ( 73 \%) consideraron Suficiente la información que se solicita sobre las actividades realizadas y casi todos tuvieron una opinión favorable de la utilidad de los datos que se obtienen del parte diario; no obstante, entre los comentarios del $5 \%$ que la consideraron No útil estuvo:

...no nos enteramos de lo que se procesa, no recibimos información de los resultados y no participamos de los análisis, no creemos que sea útil tanta información que hasta impide cumplir con lo planificado.

Un planteamiento reiterado en las dinámicas de grupo fue la no participación del personal en el análisis de los indicadores obtenidos a partir de la información que ellos mismos generan.

En relación con el sistema de reconocimiento y estimulación a los trabajadores, una mayoría refirió que su labor sólo le es reconocida por parte del jefe inmediato y los compañeros del mismo equipo. Expresaron no sentirse reconocidos por compañeros de otros equipos, de los niveles superiores, representantes de otros sectores, del sindicato, de las organizaciones políticas, ni por la población.

Según la encuesta, el 42,2 \% opinó que Ocasionalmente son estimulados por la realización de su trabajo y el 30,7 \% expresó que Nunca. El análisis cualitativo demostró la existencia de irregularidades en el pago, no correspondencia entre las horas trabajadas y el estímulo establecido. Una expresión muy frecuente encontrada como respuesta a la pregunta abierta del cuestionario fue: "... Jos indicadores de la estimulación fueron diseñados como para no ser pagados nunca". En las dinámicas grupales se repitieron cuestiones como estas:

...no nos sentimos estimulados ni reconocidos a ningún nivel de la dirección, necesitamos estar representados en el sindicato pues las condiciones de emulación con los otros profesionales no son las mismas y nosotros estamos en desventaja, la atención al hombre no es igual para todos; para el personal de apoyo hay un trato diferente, ellos reciben mejor atención.

Ante la pregunta, ¿Considera Ud. que tiene dificultades para realizar su trabajo?, el $42,6 \%$ de los trabajadores respondió que Sí, y expresaron que estas estaban relacionadas mayormente con los recursos y el apoyo para la realización de las 
tareas seguido en orden decreciente de frecuencia por problemas de coordinación, capacitación, funcionamiento y dirección.

A partir del análisis de la información proveniente del inventario de clima, este se consideró no favorable (tabla 3 ).

Tabla 3. Resultados de los criterios evaluativos del clima organizacional según dimensiones

\begin{tabular}{|c|c|c|c|}
\hline Dimensiones & Criterios & Resultados & Evaluación \\
\hline \multirow{4}{*}{ Liderazgo } & Dirección & 2,42 & Satisfactorio \\
\hline & Estímulo a la excelencia & 2,22 & Satisfactorio \\
\hline & Estímulo al trabajo en equipo & 2,15 & Satisfactorio \\
\hline & Solución de conflictos & 2,13 & Satisfactorio \\
\hline \multirow{4}{*}{ Motivación } & Realización personal & 2,13 & Satisfactorio \\
\hline & Reconocimiento a la aportación & 1,45 & Insatisfactorio \\
\hline & Responsabilidad & 1,36 & Insatisfactorio \\
\hline & $\begin{array}{l}\text { Adecuación de las condiciones de } \\
\text { trabajo }\end{array}$ & 1,54 & Insatisfactorio \\
\hline \multirow{4}{*}{ Reciprocidad } & Aplicación del trabajo & 2,12 & Satisfactorio \\
\hline & Pertenencia institucional & 2,38 & Satisfactorio \\
\hline & Retribución & 1,47 & Insatisfactorio \\
\hline & Equidad & 1,74 & Insatisfactorio \\
\hline \multirow[t]{4}{*}{ Participación } & $\begin{array}{l}\text { Compromiso con la } \\
\text { productividad }\end{array}$ & 1,95 & Insatisfactorio \\
\hline & Compatibilidad de intereses & 2,05 & Insatisfactorio \\
\hline & Intercambio de la información & 1,78 & Insatisfactorio \\
\hline & Involucración con el cambio & 1,67 & Insatisfactorio \\
\hline
\end{tabular}

El Liderazgo, entendido como la influencia ejercida por los jefes en el comportamiento de sus subordinados, por medio de la orientación y el convencimiento para ejecutar con entusiasmo las actividades asignadas y alcanzar mejores resultados de trabajo, fue la única dimensión que tuvo un comportamiento favorable, pese a que todos los criterios estuvieron muy cerca del estándar establecido. Este resultado no fue coincidente con la respuesta a la pregunta abierta del cuestionario ni con los criterios emitidos en los grupos focales.

Por el contrario, los resultados en la dimensión Participación hablaron a favor de una organización que no se desenvuelve bajo un clima laboral favorable. Ninguno de los criterios estudiados en esta dimensión que expresa la contribución de los trabajadores al logro de los objetivos reveló resultado satisfactorio, aunque el referido a la correspondencia de los objetivos del colectivo con los del departamento y el interés porque estos se cumplan (compatibilidad de intereses), alcanzó un valor muy cerca del estándar.

La Realización personal, expresada en el nivel de importancia que los trabajadores confieren a su actividad y el sentimiento de orgullo por pertenecer al colectivo, fue el único criterio en la dimensión Motivación que se comportó satisfactoriamente, aunque también muy cerca del valor mínimo aceptado.

Por otra parte, en la dimensión Reciprocidad, interpretada como la relación trabajador-organización para dar y recibir mutuamente, estuvieron afectados dos criterios: Retribución y Equidad. 
El recuadro muestra un grupo de elementos del clima organizacional en los que se expresa la influencia negativa de las dimensiones que resultaron afectadas (recuadro).

Recuadro. Elementos del clima organizacional en los que se expresa la influencia negativa de las dimensiones afectadas

\begin{tabular}{|c|}
\hline Motivación \\
\hline $\begin{array}{l}\text { - Reconocimiento a los mejores desempeños. } \\
\text { - Posibilidades para la toma de decisiones en pro de realizar un mejor trabajo. } \\
\text { - Reconocimiento por parte de los directivos de las ideas que da el colectivo. } \\
\text { - Satisfacción por las condiciones de trabajo en el orden de infraestructura y el ambiente } \\
\text { psicosocial. }\end{array}$ \\
\hline Reciprocidad \\
\hline $\begin{array}{l}\text { - Oportunidades de superación y capacitación, así como disponibilidad de planes de } \\
\text { desarrollo para los trabajadores. } \\
\text { - Objetividad y valía de las promociones como mecanismo de premiación al mejor } \\
\text { desempeño. }\end{array}$ \\
\hline Participación \\
\hline $\begin{array}{l}\text { - Correspondencia y cumplimiento armónico de los objetivos del grupo con los del } \\
\text { - Opartamento. } \\
\text { - Fluidez y oportunidad de las informaciones requeridas para cumplir con la función. } \\
\text { - Unidad entre los diferentes grupos de trabajo aun cuando participen como equipo ante } \\
\text { retos que se presentan. } \\
\text { - Idoneidad del personal. }\end{array}$ \\
\hline
\end{tabular}

\section{DISCUSIÓN}

Hoy en día existe un renovado interés por las organizaciones y el capital humano, de ahí que explorar los aspectos organizacionales que afectan el desempeño, va más allá del estudio de la efectividad y la eficiencia en el trabajo, para adentrarse en aquellos aspectos que tienen trascendencia en la conducta y comportamiento de las personas que laboran en una institución, como es el clima organizacional, entendido como la percepción que los miembros de una organización tienen de las características más inmediatas que les son significativas, que la describen y diferencian de otras organizaciones, o en otros términos, las percepciones que el trabajador tiene de las estructuras y procesos que ocurren en su medio laboral, según lo define Alexis Goncalves, citado por Bustos $P,{ }^{5}$ una persona que ha dedicado su vida profesional a investigar este tema.

Los elementos relativos a la estructura y funcionamiento de una institución además de determinar su clima, en función de las percepciones de sus miembros, inducen determinados comportamientos y conductas que inciden en el desempeño de la organización. Se dice que en el clima influyen todos los factores objetivos y subjetivos que están presentes en las instituciones, las formas y condiciones de trabajo, los estilos de dirección, los mecanismos de reconocimiento, las relaciones interpersonales, la cooperación, la comunicación y otros. ${ }^{6}$

Demandados por la velocidad, novedad, profundidad y amplitud en el enfoque de funcionamiento organizacional según necesidades específicas de cada momento, se 
han producido cambios significativos en la actividad de Dirección; en particular los cambios en el entorno y en la concepción de los recursos humanos, ${ }^{7}$ por lo que el logro de la adaptación de las instituciones de salud a estas demandas es sin dudas un reto para quienes la dirigen.

En salud cada organización tiene elementos que la distingue y las diferencia unas de otras, de igual modo, cada grupo de personas desarrolla características especiales, ingredientes de la cultura del grupo y de la institución.

Las difíciles situaciones, objetivas y subjetivas, que de forma exitosa tiene que enfrentar el Área de Higiene y Epidemiología hace complejo el accionar de sus instituciones y de manera particular el de los Grupos de Control de Vectores, lo que al mismo tiempo, los hace menos factible de ser manejados con estilos de dirección "tradicionales". Se impone la adopción de nuevos estilos en los que las formas de trabajo y diseño de procesos sean más participativos; apoyados en el conocimiento que tienen todas las personas y su socialización, de tal manera, que en el equipo de trabajo se fomente el intercambio de opiniones e ideas, las relaciones entre compañeros y con ello, el entendimiento y aceptación de orientaciones y estrategias que tienen como único fin resolver los problemas.

Cada día son más los defensores del estilo de mando participativo en el que se valora tanto la tarea como el individuo y en el que la función de dirección queda centrada en la coordinación de los miembros del grupo, de igual modo, la implementación de estrategias de mejoras que fomentan, a través de la formación, autonomía y motivación, el deseo de conocer, saber y hacer en los trabajadores. ${ }^{8}$

Ello precisa, entre otros elementos, del conocimiento de los trabajadores de la misión de su institución, del establecimiento de un sistema de comunicación efectivo que facilite el intercambio de información en todas las direcciones, con uso de un lenguaje que pueda ser compartido por todos los miembros y que facilite la cooperación a todos los niveles; una comunicación en la que prevalezca la retroalimentación sobre el trabajo realizado, tanto positivo como negativo, con lo que se crea una cultura organizacional en la que primen los valores y el compromiso.

No puede funcionar bien una organización donde no sean precisas y efectivas las coordinaciones y la comunicación que deben existir para el trabajo, tanto intra como extrasectorial y donde además, no fluya la información, tanto vertical como horizontal, que garantice la posibilidad de perfeccionamiento de los mecanismos creados.

Los factores organizacionales existentes, externos y principalmente internos, influyen sobre el desempeño de los miembros dentro de la organización y dan forma al ambiente en que esta se desenvuelve, pero no influyen directamente, sino sobre las percepciones que sus miembros tengan de cada uno de estos factores; por tanto, el comportamiento de una persona, miembro de la organización, no es sino el resultado de las percepciones que tenga de cada uno de estos factores, que dependen en buena medida de las actividades, interacciones y otra serie de experiencias que cada cual tenga de la organización. De ahí que el clima refleja la interacción entre características personales y organizacionales. ${ }^{5}$

Coincidiendo con otros estudiosos del tema, ${ }^{9}$ en ocasiones se trata de explicar por qué algunos Grupos de Control de Vectores obtienen buenos resultados en su desempeño pese a funcionar también en condiciones difíciles, con condiciones higiénico-sanitarias poco favorables en su entorno y limitados recursos. Quizás la 
respuesta sea que ese personal está motivado y seriamente comprometido con la tarea, creen en lo que hacen, se sienten atendidos, participan en las decisiones y generan ideas que llevan al logro de esos resultados positivos.

Con frecuencia, las insuficiencias en el trabajo de la Campaña, han sido encaradas mediante cambios en la estructura y formas organizativas, procesos capacitantes e incentivos monetarios como una expresión de mejoramiento de la "atención al hombre", sin estudiar a fondo la situación organizacional* que influye, entre otros, en la estabilidad del personal, la calidad del trabajo y el despeño de los grupos en general.

Está probado que los llamados "sistemas de recompensas", en todas sus variantes, condicionan conductas más favorables en el proceso de trabajo, además de incrementar el nivel de compromiso de los trabajadores con el cumplimiento de los objetivos de la organización, ${ }^{7}$ por ello el reconocimiento y la formación de aptitudes, actitudes y conocimientos de este personal, cuyas labores se realizan en medio de la escasez de recursos, en irregulares condiciones de trabajo y con bajo reconocimiento social por parte de la población, son aspectos vitales; pero sin olvidar que la capacitación, los cambios de estructuras y la estimulación, incluso moral, por si solos no resultan efectivos si existen problemas que siendo percibidos por los trabajadores no son investigados para su posible solución.

Son casi nulos los trabajos publicados en el Área de Higiene y Epidemiología que revelen esta temática lo que hace difícil contrastar los resultados del presente estudio. Algunos de ellos difieren de otros estudios realizados en la Escuela Nacional de Salud Pública (ENSAP), pero resultan similares a los encontrados por Fernández de la Nuez y Morales Sánchez. ${ }^{10,11}$

Un resultado no esperado en el estudio, fue el comportamiento que tuvo la dimensión Liderazgo atendiendo a las opiniones que dieron los trabajadores en los grupos focales, lo que pudiera estar dado en que la mayoría de los encuestados eran operarios cuya opinión sobre los directivos se refirió a sus jefes de brigada, con los que existía un mayor «compromiso» de apoyo mutuo, en medio de un escenario en que la autoridad estaba delegada hacia otros niveles por acontecer una epidemia.

Al igual que en las referidas investigaciones, un importante número de trabajadores no tenían "idea" de cual es la razón de ser de la vicedirección a la que pertenecen, de la que son parte y a la que deben contribuir con un rol específico, con funciones desagregadas en tareas y actividades concretas, más aún, cuando la totalidad de los directores y una buena parte de los vicedirectores en las áreas de salud estudiadas, habían cursado el diplomado I de Dirección en Salud el que culmina con un proyecto de transformación estratégica. La misión es una expresión de la manera que las personas ven la organización y una motivación inspiradora para el trabajo.

Finalmente se puede concluir que con el empleo de técnicas cuantitativas y cualitativas y su triangulación se identificaron elementos organizacionales que caracterizaron la situación existente en una muestra de Grupos de Control de Vectores en la provincia Ciudad de La Habana y aunque la metodología empleada no permite hacer una generalización a todas las áreas de salud, fue indiscutible la existencia de numerosos problemas percibidos por los trabajadores en el orden de recursos, apoyo, coordinación, capacitación, funcionamiento y dirección; que obstaculizaban la dinámica de trabajo e influían negativamente en el clima de los grupos. 


\section{REFERENCI AS BI BLI OGRÁFI CAS}

1. Del Puerto C, Ferrer H, Toledo G. Higiene y Epidemiología. Apuntes para la Historia. La Habana: Palacio de las Convenciones; 2002.

2. Ministerio de Salud Pública de Cuba. La integración de la higiene y la epidemiología a la Atención Primaria de Salud. La Habana: MINSAP; 1995.

3. Ministerio de Salud Pública de Cuba. Dirección de Servicios Ambulatorios. Manual de Organización del Policlínico. La Habana: MINSAP; 2005.

4. Jiménez Cangas L. Modelo de integración docencia-servicio para el cambio organizacional en instituciones de salud [tesis]. La Habana: Facultad de Salud Pública; 1998.

5. Bustos P. Clima organizacional [sitio de Internet]. 2002 [citado 27 Sept 2008]. Disponible en:

http://www.gestiopolis.com/recursos/documentos/fulldocs/rrhh/clio.htm

6. Sandoval Caraveo MC. Concepto y dimensiones del clima organizacional. Hitos de Ciencias Económico Administrativas. 2004;27:78-82.

7. Carnota Laussán O. El cambio hacia el alto desempeño [CD-ROM]. La Habana: MINSAP;2004. [Biblioteca virtual para formación postgraduada de directivos del sector salud].

8. Heras Alonso T. De la organización burocrática, a la organización que aprende y reconoce las competencias [sitio de Internet]. 2002 [citado 3 Ene 2007]. Disponible en:

http://www.monografias.com/trabajos902/organizacion-burocraticacompetencias/organizacion-burocratica-competencias.shtml

9. Lusthaus Ch. Evaluación Organizacional: marco para mejorar el desempeño. Ottawa: Centro Internacional de Investigaciones para el Desarrollo; 2002.

10. Morales Sánchez I. Diagnóstico organizacional en las vicedirecciones de Higiene y Epidemiología. Policlínico "28 de enero"del municipio Playa [tesis]. La Habana: Facultad de Salud Pública; 1999.

11. Fernández de la Nuez O. Estrategia de Perfeccionamiento Organizacional para las Vicedirecciones de Higiene y Epidemiología de Policlínicos. Municipio Playa [tesis]. La Habana: Facultad de Salud Pública; 1999.

Recibido: 11 de noviembre de 2008.

Aprobado: 23 de noviembre de 2008. 
Vivian Noriega Bravo. Escuela Nacional de Salud Pública. Calle Línea esq. I. El Vedado. La Habana 10400, Cuba.

E-mail: vivy@infomed.sld.cu

*Situación organizacional, entendida como el estado, en un momento dado, de la estructura y otras características de la organización que son más menos

permanentes en el tiempo, y la percepción que de ello tienen los trabajadores. 